\title{
A driftless gas proportional scintillation counter with a P10 microstrip photosensor in a 5-T magnetic field
}

\author{
J.F.C.A. Veloso ${ }^{\mathrm{a}, *}$, C.A.N. Conde ${ }^{\mathrm{a}}$, O. Huot ${ }^{\mathrm{b}}$, P. Knowles ${ }^{\mathrm{b}}$, F. Kottmann ${ }^{\mathrm{c}}$, \\ F. Mulhauser ${ }^{\mathrm{b}}$, J.M.F. dos Santos ${ }^{\mathrm{a}}$, D. Taqqu ${ }^{\mathrm{d}}$ \\ ${ }^{a}$ Departamento de Física, Universidade de Coimbra, 3004-516 Coimbra, Portugal \\ ${ }^{\mathrm{b}}$ Institut de Physique de l'Université, CH-1700 Fribourg, Switzerland \\ ' Institut für Teilchenphysik, ETHZ, CH-8093 Zürich, Switzerland \\ ${ }^{\mathrm{d}}$ Paul Scherrer Institut, CH-5232 Villigen-PSI, Switzerland
}

Received 19 November 2001; received in revised form 23 January 2002; accepted 24 February 2002

\begin{abstract}
The operating characteristics of a xenon driftless gas proportional scintillation counter with a P10 microstrip gas chamber photosensor in magnetic fields up to $5 \mathrm{~T}$ are described. Energy resolution of $19 \%$ for $2.3-\mathrm{keV}$ X-rays and an equivalent noise of $<50 \mathrm{eV}$ were obtained. Pulse amplitude and energy resolution were unaffected from 0 to $5 \mathrm{~T}$, demonstrating the insensitivity of this type of detector to strong magnetic fields. (C) 2002 Elsevier Science B.V. All rights reserved.
\end{abstract}

Lamb-shift spectroscopy in muonic hydrogen [1] requires a large area $2-\mathrm{keV} \mathrm{X}$-ray detector capable of operating in a 5-T magnetic field. This involves stopping $\mu^{-}$in low-pressure hydrogen to form muonic hydrogen in the $2 \mathrm{~S}$ metastable state. Muonic hydrogen is then elevated to the $2 \mathrm{P}$ state by a pulsed laser beam and subsequently decays to the ground state with the emission of a $1.9-\mathrm{keV}$ photon. The $2 \mathrm{~S}-2 \mathrm{P}$ energy difference can be determined by measuring the number of coincidences between the laser pulse and the $1.9-\mathrm{keV}$ photons as a function of the laser wavelength. An accurate measurement of the equivalent Lamb Shift in muonic hydrogen is a stringent test of quantum electrodynamics and the rms proton

\footnotetext{
*Corresponding author. Tel.: + 351-239410667; fax: +351239829158.

E-mail address: jveloso@gian.fis.uc.pt (J.F.C.A. Veloso).
}

radius can be determined to better than 1 part in 1000. To achieve this accuracy, a detector with an energy resolution of better than $20 \%$ for $1.9-\mathrm{keV}$ $\mathrm{X}$-rays, a large detection area $\left(20 \times 4 \mathrm{~cm}^{2}\right)$, timing resolution better than $100 \mathrm{~ns}$, and good behaviour in a 5-T magnetic field is required. Indeed, future particle physics experiments already urgently need $\mathrm{X}$-ray detectors capable of operating in strong magnetic fields, e.g. [2].

The potential uses of a driftless gas proportional scintillator counter (GPSC) capable of operating in strong magnetic fields by the substitution of a CsI-coated microstrip plate (MSP) photosensor in place of a photomultiplier tube has been recognized [3]. Although good-operating characteristics are expected, the actual performance has been limited by the optical positive feedback produced in the avalanche process that occurs near the MSP anodes in a xenon atmosphere. As a result, the 


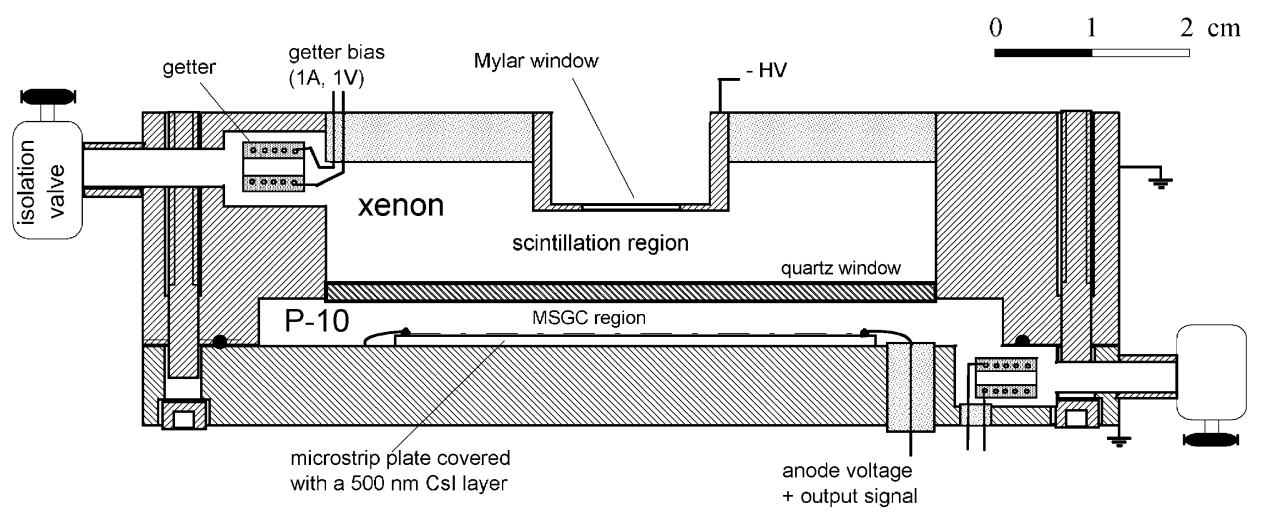

Fig. 1. Schematic of the proposed GPSC/P10-CsI-MSP X-ray detector built for the present work.

gain of the system is compromised, as are the energy and time resolutions. To overcome this limitation, a 2-keV X-ray detector based on a xenon driftless GPSC using a CsI-covered MSP in a P10 atmosphere as the VUV photosensor [4,5], was proposed.

The detector, shown in Fig. 1, consists of a 6mm-deep xenon scintillation region separated from the P10 photosensor chamber by a $1-\mathrm{mm}$ quartz window. An MSP (CERN MS-4 model, $30 \times 30 \mathrm{~mm}^{2}$ effective area, on a $500-\mu \mathrm{m}$ Desag D263 glass substrate) is placed in the $\mathrm{P} 10$ chamber, $3 \mathrm{~mm}$ away from the quartz window and is coated with a 500-nm CsI film. Gas purification in both chambers is achieved with small (1 A, 1 W) resistive getters (SAES St 172). These features result in a very compact and portable detector. All materials used for the chamber are non-ferromagnetic, e.g. Macor, stainless steel (316 L), and the magnetic field is therefore affected on a relative level below $10^{-3}$ which is negligible for our purpose.

Incident soft X-rays are absorbed in xenon by photoelectric effect near the entrance window and produce a primary electron cloud. In the scintillation region the electrons are accelerated by an electric field chosen to be above the xenon scintillation threshold (i.e. above $1 \mathrm{~V} \mathrm{~cm}^{-1} \mathrm{Torr}^{-1}$ ). The excited xenon excimers produce ultraviolet $(170 \mathrm{~nm})$ luminescence photons that are transmitted through the quartz window. Upon reaching the MSP, the UV photons eject photoelectrons from the CsI-covered microstrip cathodes. These photoelectrons drift towards the anodes where they undergo multiplication in the P10 gas due to the high electric field present in the anode region. The charge collected by the anode strips is proportional to the energy of the incoming X-rays absorbed in the xenon volume of the GPSC within the statistical fluctuations inherent to the detection and amplification processes. Since P10 does not scintillate there is no light produced in the P10 chamber reaching the CsI-covered microstrip and so the optical positive feedback effects observed if the chamber was filled with xenon, that deteriorate performance [3,5], are eliminated.

In principle this arrangement should not depend on the magnetic field. Fig. 2 shows the detector orientation relative to the electric $\vec{E}$ and magnetic $\vec{B}$ field. For the same conditions, we concluded in Ref. [3] that the effect of the magnetic field on the electron energy distribution and, thus on the GPSC secondary scintillation yield, is negligible.

In addition, for the same conditions, the Lorentz angle, $\alpha_{\mathrm{L}}$, does not depend much on the magnetic field, since for a typical reduced electric field $E / p=5 \mathrm{~V} \mathrm{~cm}^{-1} \mathrm{Torr}^{-1}$, the electron drift

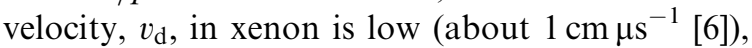
leading to

$\alpha_{\mathrm{L}}=\operatorname{arctg}\left(\frac{\left|\vec{v}_{\mathrm{d}} \times \vec{B}\right|}{|\vec{E}|}\right) \cong 5^{\circ}$.

For a 5-T magnetic field this value is too small to have a significant effect on light collection due to 


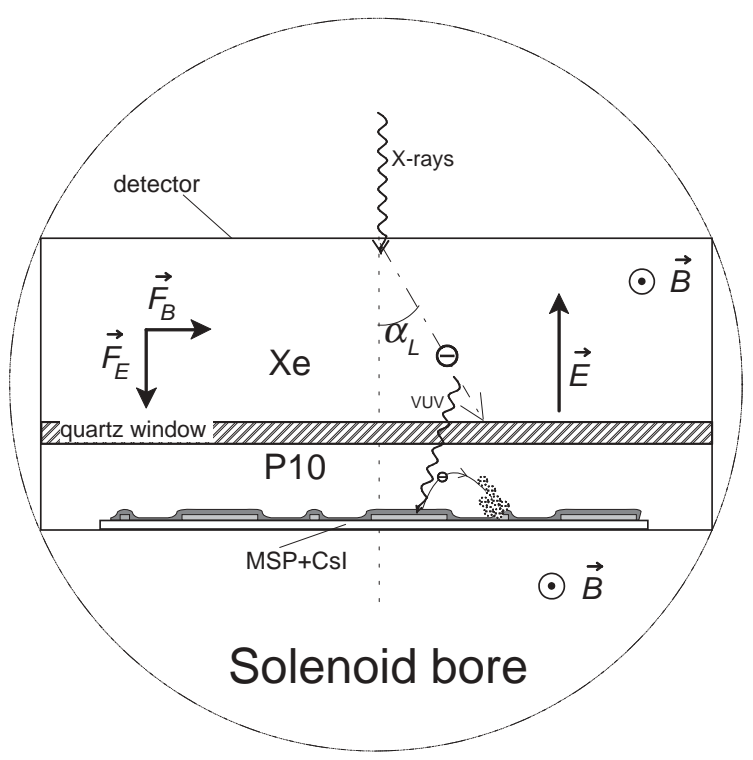

Fig. 2. Detector and electromagnetic fields geometries and orientation for the experimental set-up of the performance of the detector under strong magnetic fields.

any solid-angle effects. This is because the maximum deviation from the vertical axis is only approximately $0.45 \mathrm{~mm}$ after drifting across the full $6 \mathrm{~mm}$ long scintillation region.

Another factor that could lead to magnetic-field sensitivity is the CsI photocathode quantum efficiency (QE). Photoelectrons leaving the photocathode follow a cycloid trajectory under the effects of the Lorentz force and can return to the photocathode surface [3]. On the other hand, no variation in the MSP gain with magnetic field was noticed in previous works [7]. As we shall see from the experimental results, the effect of these contributions should be negligible.

Using a $1.5 \mathrm{~V} \mathrm{pC}^{-1}$ charge-integrating preamplifier and a spectroscopy amplifier with a $500 \mathrm{~ns}$ shaping time, a sulphur target was irradiated with a ${ }^{55} \mathrm{Fe} X$-ray source to produce $2.3-\mathrm{keV}$ X-rays. For these tests the xenon chamber was pressurized to 1200 Torr $^{1}$, with the P10 chamber at 800 Torr. The reduced electric field in the scintillation region was $E / p=7 \mathrm{~V} \mathrm{~cm}^{-1} \mathrm{Torr}^{-1}$ and the anode-tocathode MSP voltage was $V_{\mathrm{a}}=440 \mathrm{~V}$.

\footnotetext{
${ }^{1} 1 \mathrm{Torr}=133 \mathrm{~Pa}$.
}

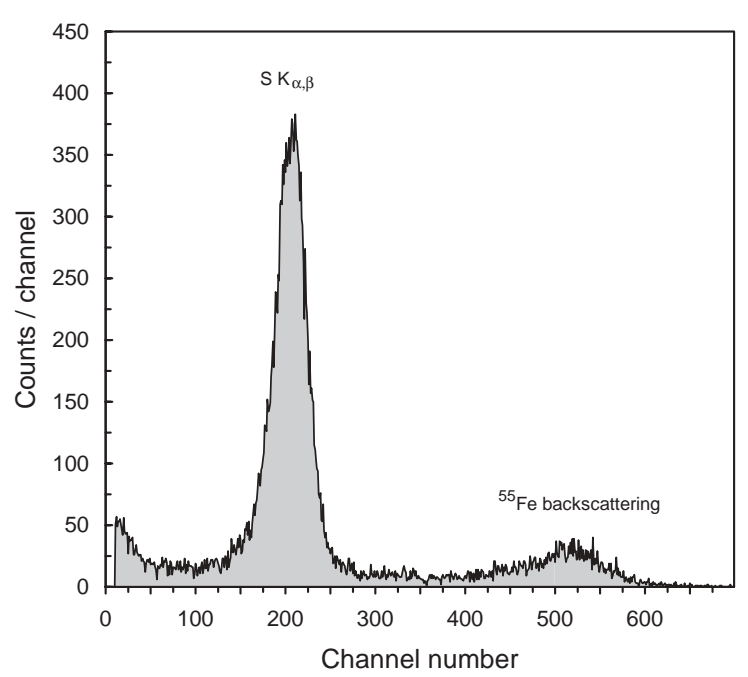

Fig. 3. X-ray fluorescence pulse-height spectrum for a sulphur $\left(2.3 \mathrm{keV} \mathrm{K}\right.$-line) target exited with a ${ }^{55} \mathrm{Fe} \mathrm{X}$-ray source.

The pulse-height distribution obtained for these conditions is shown in Fig. 3. The spectral features include the sulphur K-line, X-rays backscattered from the source, and the tail due to electronic noise. The low-energy detection limit due to electronic noise is $<50 \mathrm{eV}$. Energy resolutions of $19 \%$ is obtained for $2.3 \mathrm{keV} \mathrm{X}$-rays (sulphur Kline). The same energy resolution was achieved for $1.7 \mathrm{keV} \mathrm{X}$-rays obtained by irradiation of a silicon target with a ${ }^{55} \mathrm{Fe} \mathrm{X}$-ray source. These values compare favourably with the $22 \%$ and $25 \%$ obtained with a driftless GPSC with a CsI-covered MSP placed within the xenon scintillation gas [3] and a xenon filled microstrip gas chamber (MSGC) [8]. Additionally, signal-to-noise ratio for $2.3 \mathrm{keV}$ was improved from 7.7 [3] and 11.5 [8] to about 50 .

Tests in the magnetic field were carried out at the Paul Scherrer Institute (PSI), Switzerland, using a 1-m-long and 20-cm-bore-diameter superconducting solenoid, capable of achieving magnetic fields up to $5 \mathrm{~T}$, uniform $\left(10^{-4}\right)$ over an axial distance of $30 \mathrm{~cm}$ from the centre. The detector pulse amplitude was monitored as a function of magnetic field for $2.3-\mathrm{keV} \mathrm{X}$-rays and for a reduced electric field of $4.5 \mathrm{~V} \mathrm{~cm}^{-1} \mathrm{Torr}^{-1}$ in the scintillation region, with the MSP $V_{\mathrm{a}}$ voltage at $360 \mathrm{~V}$ (charge gain $\sim 600$ ). 


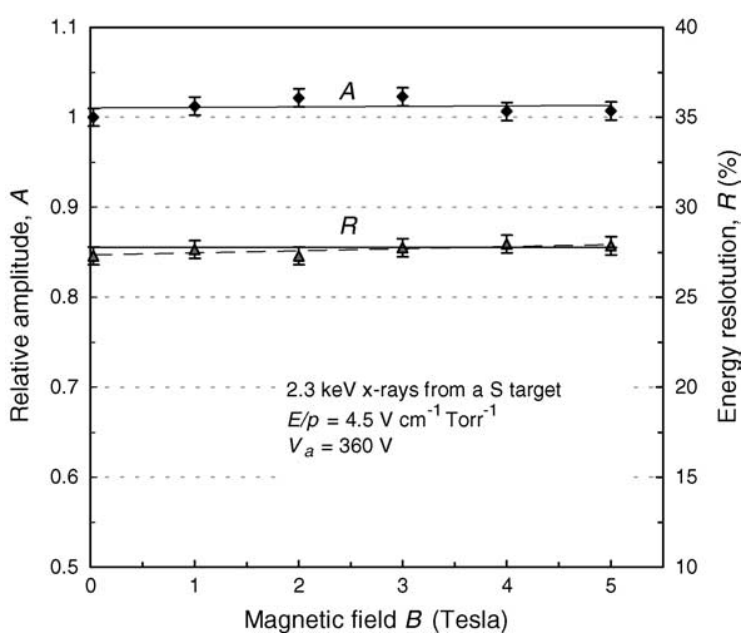

Fig. 4. Variation of pulse amplitude $(A)$ and energy resolution $(R)$ for $2.3-\mathrm{keV} \mathrm{X}$-rays as a function of magnetic field intensity.

The detector orientation inside the solenoid was chosen such that the MSP electrodes were parallel to the magnetic field direction (Fig. 2). This is the most unfavourable orientation for CsI-MSP operation since the photoelectrons drift towards the strip anodes along a direction perpendicular to the magnetic field.

Data were obtained varying the magnetic field intensity inside the solenoid in 1-T steps up to $5 \mathrm{~T}$. Fig. 4 shows the relative pulse-height amplitude of the 2.3-keV X-ray centroid as a function of magnetic field intensity. A maximum variation of less than $2.3 \%$ was obtained, which is within the experimental error. The poor energy resolution obtained during the tests in the solenoid $(\sim 28 \%)$ is due to the use of low-voltage settings to prevent any possibility of MSP damage. Non-variation of the energy resolution was observed. Thus, no degradation in the detector performance was observed, in agreement with the estimates of the magnetic field effects mentioned above. Additionally, the electronic noise tail did not change with the magnetic field.

For comparison we present in Fig. 5 the pulseheight variation with $B$ for the present detector (a) $(2.3 \%)$ together with the results obtained for the same conditions in a xenon driftless GPSC with the CsI-covered MSP placed within the xenon scintillation gas (b) (25\%) [3]. The backscattering

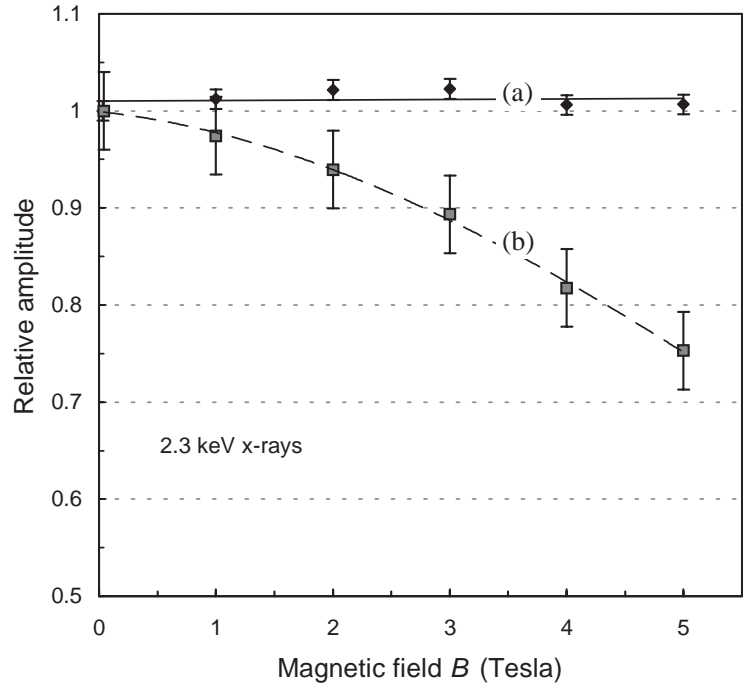

Fig. 5. Comparison of the pulse amplitude variation as a function of magnetic field for the present detector (a) and for an earlier version (b) [3] with the CsI-MSP operating within the xenon envelop.

in a P10 atmosphere is much less than that in xenon $[9,10]$, favouring both photoelectron collection efficiency and reduced sensitivity to magnetic field. Additionally, the higher electric field intensity at the MSP cathode surface for the P10 version results in longer photoelectron cycloid paths and thus smaller sensitivity to magnetic fields [3].

The detector time resolution was measured at $0 \mathrm{~T}$, using a ${ }^{54} \mathrm{Mn}$ radioactive source placed between the GPSC and an $\mathrm{NaI}(\mathrm{Tl})$ detector. The source emits $\mathrm{Cr} \mathrm{X}$-rays in coincidence and in opposite directions with $800-\mathrm{keV} \gamma$-rays. The reference signal was generated by the $800-\mathrm{keV} \gamma-$ rays, detected by the $\mathrm{NaI}(\mathrm{Tl})$. The timing signals were fed through timing filter amplifiers and constant fraction discriminators through the START and STOP inputs of a TDC. Detecting the 5.4-keV X-rays, with the GPSC, in coincidence with the $800-\mathrm{keV} \gamma$-rays, a time resolution of about $40 \mathrm{~ns}$ (FWHM) was measured for this system. Thus, the GPSC/MSGC-P10 detector fulfills the timing requirements for the referred experiment.

We have shown that a xenon driftless GPSC using as photosensor a P10 filled chamber with a MSP covered with CsI, presents good performance 
characteristics for the detection of low energy $\mathrm{X}$-rays (19\% energy resolution for 2.3 and $1.7 \mathrm{keV}$ $\mathrm{X}$-rays, and a minimum detection limit of $50 \mathrm{eV}$ ) and can operate under magnetic fields up to $5 \mathrm{~T}$ with no detectable performance degradation.

The detector development work was carried out in the Atomic and Nuclear Instrumentation Group of the Instrumentation Center (Unit 217/94) of the physics Department of University of Coimbra. The studies of the performance of this detector under strong magnetic field were carried out at the Paul Scherrer Institut, in Switzerland. Thanks are due to R.E. Morgado (LANL, USA) for manuscript revising. Support is acknowledged to Fundação para a Ciência e a Tecnologia (FCT) through Project PRAXIS/P/FIS/13140/1998, and to Swiss National Science Foundation. J.F.C.A. Veloso acknowledges support from FCT.

\section{References}

[1] D. Taqqu, et al., Hyperfine Interactions 119 (1999) 311.
[2] F. Piuz, et al., Nucl. Instr. and Meth. A 433 (1999) 178.

[3] J.F.C.A. Veloso, J.M.F. dos Santos, C.A.N. Conde, F. Mulhauser, P. Knowles, C. Donche-Gay, O. Huot, D. Taqqu, F. Kottmann, Nucl. Instr. and Meth. A 460 (2001) 297.

[4] J.F.C.A. Veloso, J.M.F. dos Santos, C.A.N. Conde, Nucl. Instr. and Meth. A 422 (1999) 273.

[5] J.F.C.A. Veloso, J.M.F. dos Santos, C.A.N. Conde, Nucl. Instr. and Meth. A 457 (2001) 253.

[6] T.H.V.T. Dias, F.P. Santos, A.D. Stauffer, C.A.N. Conde, Phys. Rev. A 48 (1993) 2887.

[7] F. Angelini, et al., Nucl. Instr. and Meth. A 343 (1994) 441.

[8] J.F.C.A. Veloso, J.M.F. dos Santos, C.A.N. Conde, R.E. Morgado, X-ray Spectrom. 26 (1997) 237.

[9] P.J.B.M. Rachinhas, J.A.M. Lopes, T.H.V.T. Dias, F.P. Santos, C.A.N. Conde, A.D. Stauffer, Photoelectron collection efficiency in rare gases: a Monte Carlo study, in: M. Nakagawa, P. Vaz, F. Barão, A. Kling, L. Távora (Eds.), Proccedings of the MC2000-International Conference on Advanced Monte Carlo for Radiation Physics, Particle Simulation and Applications, Instituto Superior Técnico, Lisboa, Portugal, 23-26 October 2000. Springer, Heidelberg, 2001, pp. 535-542.

[10] A. Di Mauro, E. Nappi, F. Posa, A. Breskin, A. Buzulutskov, R. Chechik, S.F. Biaggi, G. Paic, F. Piuz, Nucl. Instr. and Meth. A 371 (1996) 137. 